\title{
THE WARPS X-RAY SURVEY OF GALAXIES, GROUPS, AND CLUSTERS
}

\author{
D. HORNER ${ }^{1}$, C.A. SCHARF ${ }^{1}$, L.R. JONES ${ }^{2}$, H. EBELING ${ }^{3}$, \\ E. PERLMAN ${ }^{4}$, M. MALKAN ${ }^{5}$ AND G. WEGNER ${ }^{6}$ \\ ${ }^{1}$ Lab. for High Energy Astrophysics, NASA/GSFC \\ ${ }^{2}$ School of Physics and Space Research, Univ. of Birmingham \\ ${ }^{3}$ Institute for Astronomy, Univ. of Hawaii \\ ${ }^{4}$ Space Telescope Science Institute \\ ${ }^{5}$ Dept. of Astronomy, UCLA \\ ${ }^{6}$ Dept. of Physics $\mathcal{B}$ Astronomy, Dartmouth College
}

\section{Introduction}

We have embarked on a survey of ROSAT PSPC archival data searching for all detected surface brightness enhancements due to sources in the innermost $R \leq 15^{\prime}$ of the PSPC field of view in the energy band $0.5-2.0 \mathrm{keV}$. This project is part of the Wide Angle ROSAT Pointed Survey (WARPS) and is designed primarily to measure the low luminosity, high redshift, X-ray luminosity function of galaxy clusters and groups. Accurate measurements of the high redshift XLF would allow the form of the XLF evolution to be determined via the position of the Schechter function break. This would help discriminate between luminosity and density evolution, and discriminate between different hierarchical models, e.g., those including a different mix of fundamental particles, a flat power spectrum of the initial fluctuations, and reheating of the intracluster gas at high redshifts.

\section{Data Analysis}

Our source detection method, Voronoi Tessellation and Percolation or VTP, represents a significant advance over conventional methods and is particularly suited for the detection and correct quantification of extended and/or low surface brightness emission which could otherwise be missed or wrongly interpreted. We also use energy dependent exposure maps to estimate the 
fluxes of sources which can amount to corrections of as much as $15 \%$. We have nearly complete sky coverage for clusters out to a redshift of $z \simeq 1$, with $50 \%$ coverage at a redshift of $z \sim 1.4$. We have $80 \%$ coverage for groups (or faint, small clusters) to $z \sim 0.2$ and for galaxies to about $z \sim 0.1$.

In an ongoing optical follow-up program, we are obtaining both CCD imaging and spectroscopic data of the extended sources and selected pointlike VTP sources (specifically those with galaxy counterparts). The followup procedure has been designed to minimize incompleteness and misidentifications of the X-ray source candidates (catalogue in preparation). Classification based solely on X-ray criteria may be in error at low fluxes like those used in WARPS. We have found that only $70-80 \%$ of our VTP extended sources are clusters. Imaging will also allow determinations of the richnesses and morphologies of the clusters and correlation of optical and $\mathrm{X}$-ray properties of clusters.

\section{Results}

The first results for for an initial 91 fields $\left(17.2 \mathrm{deg}^{2}\right)$ at fluxes $>3.5 \times 10^{-14}$ erg $\mathrm{s}^{-1} \mathrm{~cm}^{-2}$ are presented in detail by Scharf et al. (1996). The sky density of extended objects with detected flux $>3.5 \times 10^{-14} \mathrm{erg} \mathrm{sec}^{-1}$ $\mathrm{cm}^{-2}$ is 2.8-4.0 $( \pm 0.4) \mathrm{deg}^{-2}$. A comparison with a point source detection algorithm has demonstrated that our VTP approach typically finds 1-2 more objects $\operatorname{deg}^{-2}$ to this flux limit, suggesting that the conventional method fails to detect a significant fraction of extended objects. The surface brightness limit of the WARPS cluster survey is $\sim 1 \times 10^{-15} \mathrm{erg} \mathrm{sec}^{-1}$ $\mathrm{cm}^{-2}$ arcmin $^{-2}$, approximately 6 times lower than the Extended Medium Sensitivity Survey (EMSS). The WARPS LogN-LogS (which currently represents a lower limit) shows a significant excess over previous measurements for $\mathrm{S} \gtrsim 8 \times 10^{-14} \mathrm{erg} \mathrm{sec}^{-1} \mathrm{~cm}^{-2}(0.5-2 \mathrm{keV})$. We attribute this mainly to a larger measured flux from extended sources as well as new detections of low surface brightness systems in the WARPS.

For further information and preprints of WARPS papers, see the WARPS home page at

http://lheawww.gsfc.nasa.gov/ caleb/warps/warps.html.

\section{References}

Scharf, C.A., et al. Astronhys. J., in press 